\title{
Stochastic modelling and self-tuning control of a continuous cement raw material mixing system
}

\author{
T. WESTERLUND $\dagger$, H. TOIVONEN $\dagger$ and K.-E. NYMAN $\ddagger$
}

Keywords: stochastic modelling, self-tuning control, adaptive control, computer control, stochastic control, self-adjusting systems, cement industry, chemical variables control.

The control of a continuously operating system for cement raw material mixing is studied. The purpose of the mixing system is to maintain a constant composition of the cement raw meal for the kiln despite variations of the raw material compositions. Experimental knowledge of the process dynamics and the characteristics of the various disturbances is used for deriving a stochastic model of the system. The optimal control strategy is then obtained as a minimum variance strategy. The control problem is finally solved using a self-tuning minimum variance regulator, and results from a successful implementation of the regulator are given.

\section{Notation}

$A(z)=$ polynomial defined by eqn. (2.2)

$a_{i}=$ system parameter

$B(z)=$ polynomial defined by eqn. (2.2)

$b_{t}=$ system parameter

$C(z)=$ polynomial defined by eqn. (2.2)

$c_{i}=$ system parameter

$e=$ white noise, $N(0, \sigma)$

$F(z)=$ polynomial defined by eqn. (2.6)

$G(z)=$ polynomial defined by eqn. (2.6)

$h=\beta_{0}{ }^{-1}$

$\boldsymbol{K}=$ vector of feedback gains

$\hat{\boldsymbol{K}}=$ estimated vector of feedback gains

$k=$ discrete time delay

$k_{i}=$ feedback gain

$\hat{k}_{t}=$ estimated feedback gain

$L=$ time delay in the silo model, eqn. (4.11) or (5.2)

$\boldsymbol{P}=$ normalized covariance matrix, eqn. (2.11)

$q^{-1}=$ backward shift operator

$r(t)=$ autocovariance function, eqn. (4.9)

$s^{2}=$ estimated variance

$T=$ time constant

$t=$ discrete time

$u=$ system input, eqn. (2.1)

$V=$ accumulated loss

† Department of Chemical Engineering, Åbo Akademi, SF-20500 Åbo, Finland.

\$ Oy Partek Ab, Pargas, Finland.

Part of T. Westerlund's work was done at The Division of Engineering Cybernetics, The Norwegian Institute of Technology, Trondheim, Norway. 
$v=$ drifting disturbance defined by eqn. (4.4)

$v=$ vector defined by eqn. (2.9)

$w=$ stochastic disturbance defined by eqn. (4.7)

$X=$ silica ratio

$Y=$ lime saturation factor

$Y_{\mathrm{Sp}}=$ set point for lime saturation factor

$y=$ lime saturation factor, deviation from set point, $Y-Y_{\mathrm{Sp}}$; system output, eqn. (2.1)

$y_{A}, y_{B}, y_{C}=$ measured lime saturation factor at various parts of the system, cf. Fig. $1(a)$

$y_{A}^{\prime}, y_{B}^{\prime}, y_{C}^{\prime}=$ actual lime saturation factor at various parts of the system used in simulations, cf. Fig. 1 (a)

$\hat{y}_{C}=$ estimated silo output given by eqn. (5.2)

$\boldsymbol{Z}=$ alumina ratio

$\alpha=$ model parameter in eqns. (4.11) and (5.2)

$\alpha_{i}=$ model parameter, eqn. (2.7)

$\hat{\alpha}_{l}=$ estimated model parameter

$\beta=$ model parameter in eqns. (4.11) and (5.2)

$\beta_{l}=$ model parameter, eqn. (2.7)

$\hat{\beta}_{i}=$ estimated model parameter

$\Delta=1-q^{-1}$

$\epsilon=$ residual, eqn. (2.7)

$\lambda=$ exponential weighting constant, eqn. (2.11)

$\sigma^{2}=$ variance

\section{Introduction}

High energy costs have in recent years made the wet method of cement manufacturing less profitable and most of the plants are presently being designed for the dry process. In this process an efficient mixing of the raw material components is necessary in order to maintain a constant composition of the cement raw meal for the kiln. This usually requires large investments in homogenization equipment, which damps variations of the raw material compositions. An alternative is to use a control strategy for determining the proper feed ratios of the raw material components. There is, thus, a clear incentive to study the associated control problems and some applications of advanced methods have been reported (Hammer 1972, Keviczky et al. 1978, Lundán and Mattila 1974). The disturbances are, typically, of a stochastic nature and stochastic control theory is, therefore, well suited for the problems in question.

This paper describes the control of a continuously operating cement raw material mixing system. The system has no prehomogenization of the raw material. Instead, efficient mixing is obtained by using a feedback strategy and continuously operating homogenization silos for raw meal homogenization. In this investigation, knowledge of the process dynamics and the characteristics of the various disturbances which affect the system is used for deriving a stochastic model of the system. The regulator which minimizes the variations of the raw meal composition is then obtained as the minimum variance strategy.

The objective of the control is to maintain the desired composition of the raw meal from the continuously operating homogenization silos, despite disturbances. 
A special feature of the control problem is the fact that measurements of the silo output cannot, for practical reasons, be used for feedback. A strategy minimizing the variance of the silo input, however, does not give good control of the silo output as the slow dynamics of the silos do not damp low frequencies. Therefore, the silo output is estimated from measurements of the silo input and the regulator is designed for minimizing the variance of the estimated silo output. The silo output is estimated using a first-order model and it can be shown that the performance of the strategy is insensitive to the actual residence time of the silos.

The stochastic system model has also been used for simulation purposes and the performance of a number of regulators has been investigated. It was decided to solve the control problem using a self-tuning minimum variance regulator ( $\AA$ ström and Wittenmark 1973, Åström et al. 1977). The system model implies that a self-tuning regulator, in which three parameters are estimated, should be used. However, simulations show that, when using a regulator in which only two parameters are estimated, the loss of the transient period is considerably reduced and the asymptotic average loss is only slightly larger than that of the optimal regulator. For the studied system, the transient properties of the self-tuning regulator are important owing to the extremely long sampling interval ( 1 hour). The simulations, therefore, indicate that in practice it is probably preferable to use a self-tuning regulator in which two parameters are estimated.

Finally, a successful implementation of the self-tuning regulator is described. In the implementation, a priori knowledge of the process is used by assuming that the deterministic part of the system is constant and known. Then, only those parameters which depend on the characteristics of the disturbances, which are less well known and probably time varying, are estimated.

The paper is organized as follows. In $\S 2$, a brief description of the minimum variance strategy and the self-tuning regulator is given. The mixing system is described in $\S 3$ and a stochastic model of the system is developed in $\S 4$. In $\S 5$, the performance of a number of regulators, including the self-tuning regulator, is investigated by simulations. Finally, results from the implementation of the self-tuning regulator are given in $\S 6$.

\section{A self-tuning regulator}

In this section, a brief description of the minimum variance strategy and a selftuning regulator based on this strategy is given.

\section{The system model}

Consider a linear discrete time single-input, single-output stochastic system described by

$$
\begin{array}{r}
y(t)+a_{1} y(t-1)+\ldots+a_{n} y(t-n)=b_{0} u(t-k-1)+\ldots+b_{n-1} u(t-k-n) \\
+e(t)+c_{1} e(t-1)+\ldots+c_{n} e(t-n)
\end{array}
$$

where $y$ is the output, $u$ is the input, $\{e(t)\}$ is a sequence of independent normal $\left(0, \sigma^{2}\right)$ random variables, and $k$ is the time delay. Introduce the backward shift operator $q^{-1}$, defined by

$$
q^{-1} y(t)=y(t-1)
$$


and the polynomials

$$
\left.\begin{array}{l}
A(z)=1+a_{1} z+\ldots+a_{n} z^{n} \\
B(z)=b_{0}+b_{1} z+\ldots+b_{n-1} z^{n-1}, \quad b_{0} \neq 0 \\
C(z)=1+c_{1} z+\ldots+c_{n} z^{n}
\end{array}\right\}
$$

Equation (2.1) can then be written in the compact form

$$
A\left(q^{-1}\right) y(t)=B\left(q^{-1}\right) u(t-k-1)+C\left(q^{-1}\right) e(t)
$$

The model (2.1) or (2.3) is a canonical representation of a sampled finite dimensional single-input, single-output dynamical system with time delay and disturbances which are Gaussian random processes with rational spectral densities ( $\AA$ ström 1970).

\section{The minimum variance strategy}

In the minimum variance strategy the criterion is

$$
\min _{u(t)} E y(t+k+1)^{2}
$$

where $E \cdot$ denotes the expectation operator. The optimal strategy is then given by (Åström 1970)

$$
u(t)=-\frac{G\left(q^{-1}\right)}{B\left(q^{-1}\right) F\left(q^{-1}\right)} y(t)
$$

where the polynomials

$$
\begin{gathered}
F(z)=1+f_{1} z+\ldots+f_{k} z^{k} \\
G(z)=g_{0}+g_{1} z+\ldots+g_{n-1} z^{n-1}
\end{gathered}
$$

are then defined by the identity

$$
C(z)=A(z) F(z)+z^{k+1} G(z)
$$

The following conditions are necessary:

(1) The polynomial $B(z)$ has all zeros strictly outside the unit circle. Thus, the only non-minimum phase property of the system (2.1) is the time delay $k$.

(2) The polynomial $C(z)$ has all zeros strictly outside the unit circle. This condition is not very restrictive (cf. the spectral factorization theorem ( $\AA$ ström 1970)).

If these conditions are not satisfied, suboptimal strategies must be used (Åström 1970).

\section{A self-tuning minimum variance regulator}

Self-tuning regulators (STR) have been proposed for control of systems with unknown and possibly time-varying parameters (Åström et al. 1977). A self-tuning minimum variance regulator has been given by Åström and Wittenmark (1973). In this approach, the parameters of the model

$$
\begin{aligned}
y(t+k+1)+\alpha_{1} y(t)+\ldots+\alpha_{m} y(t-m+1)= & \beta_{0}\left[u(t)+\beta_{1} u(t-1)\right. \\
& \left.+\ldots+\beta_{l} u(t-l)\right]+\epsilon(t+k+1)
\end{aligned}
$$


where $m=n$ and $l=n+k+1$ are estimated on-line by the recursive least-squares method. The estimates $\left\{\hat{\alpha}_{i}\right\},\left\{\hat{\beta}_{i}\right\}$ are then used in the corresponding minimum variance strategy,

$$
u(t)=\frac{1}{\hat{\beta}_{0}}\left[\hat{\alpha}_{1} y(t)+\ldots+\hat{\alpha}_{m} y(t-m+1)\right]-\hat{\beta}_{1} u(t-1)-\ldots-\hat{\beta}_{l} u(t-l)
$$

In order to avoid non-identifiability due to closed-loop operation it has been suggested that the parameter $\beta_{0}$ is held constant ( $\AA$ ström and Wittenmark 1973). The parameter $\beta_{0}$ is an estimate of the system parameter $b_{0}$ in (2.1). It has, however, been shown that the choice of $\beta_{0}$ is within certain limits, not critical for the behaviour of the algorithm ( $\AA$ ström and Wittenmark 1973).

Åström and Wittenmark (1973) have shown that if the regulator has the correct structure and if the parameter estimates converge to such values, that the polynomials

$$
\hat{\alpha}_{1}+\hat{\alpha}_{2} z+\ldots+\hat{\alpha}_{m} z^{m-1} \text { and } 1+\hat{\beta}_{1} z+\ldots+\hat{\beta}_{l} z^{l}
$$

have no common factors, then the control law (2.8) converges to the minimum variance strategy (2.5) which could be determined if the system parameters were known.

As the parameter $\beta_{0}$ is held constant and is not estimated, it is convenient to rewrite the model (2.7) in the form (Toivonen 1977)

$$
u(t)=h y(t+k+1)+\boldsymbol{K v}(t)+\tilde{\epsilon}(t+k+1)
$$

where $h=\beta_{0}^{-1}$,

$$
v(t)=[y(t), \ldots, y(t-m+1), u(t-1), \ldots, u(t-l)]^{\mathrm{T}}
$$

and $\boldsymbol{K}$ is a vector of feedback coefficients,

$$
\begin{aligned}
\boldsymbol{K} & =\left[\alpha_{1} / \beta_{0}, \ldots, \alpha_{m} / \beta_{0},-\beta_{1}, \ldots,-\beta_{l}\right] \\
& =\left[k_{1}, \ldots, k_{m}, k_{m+1}, \ldots, k_{m+l}\right] .
\end{aligned}
$$

The minimum variance strategy is then simply

$$
u(t)=K v(t)
$$

The STR algorithm, based on the model (2.9), can now be summed up as follows:

(1) Estimation. At each sampling instant, determine the least-squares estimate of $\boldsymbol{K}$ in the model (2.9). Using the recursive least-squares method with exponential forgetting of past data, the estimate is obtained from the equations (Eykhoff 1974).

$$
\left.\begin{array}{c}
\hat{\boldsymbol{K}}(t+1)=\hat{\boldsymbol{K}}(t)+[u(t-k)-h y(t+1)-\hat{\boldsymbol{K}}(t) v(t-k)] \frac{\boldsymbol{v}(t-k)^{\mathrm{T}} \boldsymbol{P}(t)}{1+\boldsymbol{v}(t-k)^{\mathrm{T}} \boldsymbol{P}(t) v(t-k)} \\
\boldsymbol{P}(t+1)=\frac{1}{\lambda}\left[\boldsymbol{P}(t)-\frac{\boldsymbol{P}(t) \boldsymbol{v}(t-k) v(t-k)^{\mathrm{T}} \boldsymbol{P}(t)}{1+\boldsymbol{v}(t-k)^{\mathrm{T}} \boldsymbol{P}(t) v(t-k)}\right]
\end{array}\right\}
$$

where $\lambda$ is the exponential weighting constant.

(2) Control. Determine the control signal from

$$
u(t)=\hat{\mathbf{K}}(t) v(t)
$$


It has been demonstrated that the self-tuning minimum variance regulator has good convergence properties ( $\AA$ ström and Wittenmark 1973, Åström et al. 1977). Several successful practical applications of the regulator have been reported ( $\AA$ ström et al. 1977). These include paper machines (Borisson and Wittenmark 1974, Cegrell and Hedqvist 1975), an ore crusher (Borisson and Syding 1976), an enthalpy exchanger (Jensen and Hansel 1974), a supertanker (Källström et al. 1978), a binary distillation column (Sastry et al. 1977) and a batch cement raw material blending process (Keviczky et al. 1978).

\section{The cement raw material mixing system}

The cement raw material mixing system considered in this paper belongs to the company Oy Partek Ab and is located in Pargas, Finland. The mixing system has previously been discussed by Lundán and Mattila (1974) and Laurén (1978). A schematic picture of the mixing system is shown in Fig. $1(a)$. In this process, a flow of 190 tons/hour raw material from the quarry is ground in the ball mill. The resulting cement raw meal is fed into continuously operating homogenization silos where highfrequency variations of the raw meal composition are efficiently damped. The homogenized raw meal is then preheated and fed into the cement rotary kiln. The ball mill is fed from raw feed silos containing five raw material components of different compositions. The raw meal composition is controlled by adjusting the f $\mathrm{cd}$ ratios of the raw material components. The control problem arises from the fact that the compositions of the raw materials vary from time to time and they are not measured directly.

The quality of the raw meal is characterized by the following three quantities listed in the order of decreasing priority:

$$
\begin{array}{ll}
Y=\frac{C}{2 \cdot 8 S+1 \cdot 18 A+0 \cdot 65 F} 100 & \text { lime saturation factor } \\
X=\frac{S}{A+F} & \text { silica ratio } \\
Z=\frac{A}{F} & \text { alumina ratio }
\end{array}
$$

where

$$
\begin{aligned}
& C=\text { weight- } \% \mathrm{CaO} \\
& S=\text { weight- } \% \mathrm{SiO}_{2} \\
& A=\text { weight- } \% \mathrm{Al}_{2} \mathrm{O}_{3} \\
& F=\text { weight- } \% \mathrm{Fe}_{2} \mathrm{O}_{3}
\end{aligned}
$$

In order to obtain good control of the raw meal quality, it is important to have an efficient method for analysing the raw meal and raw material compositions. Earlier, laborious wet chemical methods were used. The process of Oy Partek Ab employs a special analysing system, Cemixan (Cement Mixing Analysis), which has been described by Lundán and Mattila (1974). In this system, the calcium and iron contents are determined by an X-ray fluorescence technique and a neutron activation technique is used for the determination of the silica and alumina contents. The time needed for sample transport, sample preparation, activation and measurement is approximately 

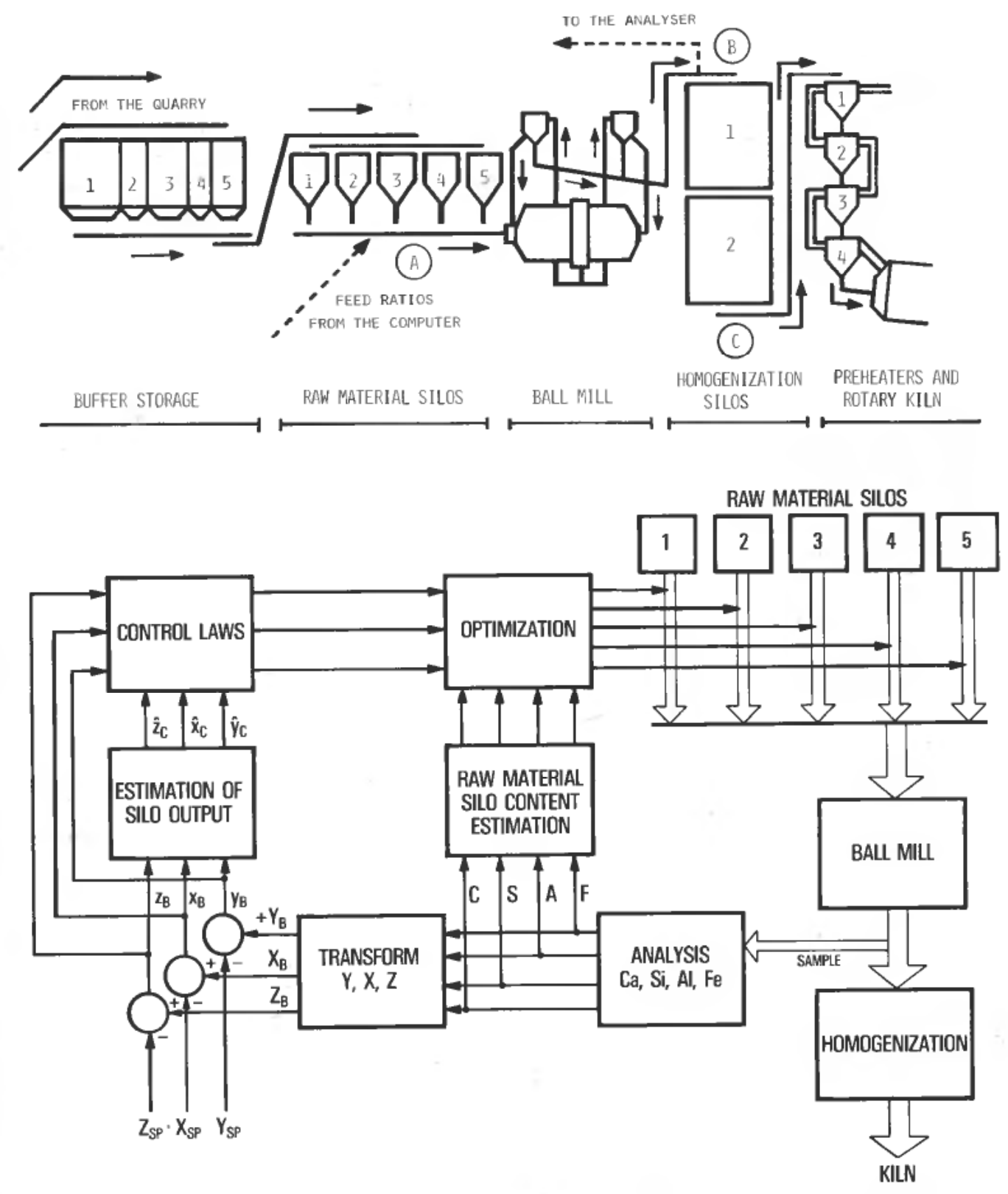

Figure I. (a) Flow sheet of the cement raw material mixing system. (b) Control configuration.

$30 \mathrm{~min}$. It has been found suitable to use a sampling period of 1 hour. The Cemixan analysing system is equipped with a PDP-11 minicomputer with $12 \mathrm{~K}$ memory, a decwriter and interfaces to the analysers and the process.

It is technically and economically impractical to obtain representative samples of the highly heterogenous raw material components in the raw feed silos. In the Cemixan system the raw material compositions are, therefore, estimated from raw meal analysis (taken at point $B$ in Fig. $1(a)$ ), and knowledge of the feed ratios (Lundán and Mattila 1974). 
The feed ratios of the raw material components are determined as follows. A control strategy generates the desired composition (lime saturation factor, silica ratio and alumina ratio) for the total feed into the ball mill during the next sampling period. The feed ratios are then determined on the basis of the estimated raw material compositions so as to generate the desired composition of the total feed. However, as there are five raw material components and only three controlled variables, there is a degree of freedom. The feed ratios are, therefore, selected by minimizing a loss function, which also puts weights on the relative costs of the raw material components (Lundán and Mattila 1974).

The control configuration of the Cemixan system is shown in Fig. I (b). The purpose of the present study is to investigate control strategies which calculate the input (desired composition of the total feed) on the basis of raw meal analysis.

\section{Stochastic modelling of the mixing system}

In this section, an approximate stochastic model of the mixing system described in $\S 3$ is developed. The dynamics of the ball mill and the homogenization silos were available from previous studies (Nyman 1976). An experimental investigation of the characteristics of the disturbances was also available (Laurén 1978).

The process is modelled so that the control signals are the desired lime saturation factor, silica ratio and alumina ratio of the total feed to the ball mill. The optimization algorithm will then calculate the proper feed ratios of the raw material components, as described in $\S 3$. The outputs are the corresponding modules of the raw meal. Now, assume that the raw material silo contents have been correctly estimated. The system can then be modelled as a decoupled multivariable system (Lundán and Mattila 1974, Nyman 1976). Thus, if for example the desired lime saturation factor (LSF) of the feed is changed, this will affect only the LSF and not the other modules of the raw meal. As the LSF is the most important criterion of the meal quality, only the model for this quantity will be discussed here.

Let the LSF at the sampling instant $t$ be denoted by $Y(t)$, and let $Y_{\mathrm{SP}}$ be the set point. It is then convenient to introduce the deviation from the set point,

$$
y(t)=Y(t)-Y_{\mathrm{SP}}
$$

It is also convenient to make a distinction between the measured quantity, $y(t)$, and the actual quantity, $y^{\prime}(t)$. These are not equivalent because of measurement errors. The LSF at a given point in the system will be indicated by a lower index; $Y_{A}, Y_{B}, Y_{C}$. (See Fig. $1(a)$.)

The dynamics of the ball mill can be described by a second-order transfer function with two equal time constants $T \approx 9 \min$ (Nyman 1976). Assume that the input to the ball mill is constant during the sampling period. The system may then be sampled. Using a sampling period equal to 1 hour and allowing for a 30 min delay in the control loop, the sampled system can adequately be described by

where

$$
y_{B}^{\prime}(t+1)=b_{0} y_{A}^{\prime}(t)+b_{1} y_{A}^{\prime}(t-1)
$$

$$
b_{0}=0.76 \text { and } b_{1}=0.24
$$

Owing to measurement errors, the measured ball mill output is

$$
y_{B}(t)=y_{B}^{\prime}(t)+e_{y}(t)
$$

where $e_{y}(t)$ is white noise with the standard deviation $\sigma_{y}=0.9$ (Laurén 1978). 
Owing to variations of the raw material compositions, there is an error in the determination of the mill input. The relationship between the desired input, $u(t)$; and the actual input to the ball mill, $y^{\prime}{ }_{A}(t)$, can approximately be given by

$$
y_{A}^{\prime}(t)=u(t)+v(t)+e_{u}(t)
$$

where $e_{u}(t)$ is white noise with the standard deviation $\sigma_{u}=8$ and $v(t)$ is a drifting disturbance, which can be characterized by

$$
v(t)=v(t-1)+e_{v}(t), \quad \sigma_{v}=1
$$

It may be observed that the sampled model (4.1) was obtained assuming that the ball mill input is constant during the sampling period. We have, thus, made the approximation that the disturbance at the input is constant during the sampling period. The limited experimental data available did not make it possible to give a more precise description of the disturbances. However, it is believed that this approximation is not too serious in comparison with other approximations made in the modelling of the system.

Eliminating the drifting disturbance $v(t)$ from eqn. (4.3) gives

$$
\Delta y_{A}^{\prime}(t)=\Delta u(t)+e_{v}(t)+e_{u}(t)-e_{u}(t-1)
$$

where

$$
\begin{gathered}
\Delta y_{A}^{\prime}(t)=y_{A}^{\prime}(t)-y_{A}^{\prime}(t-1) \\
\Delta u(t)=u(t)-u(t-1)
\end{gathered}
$$

Equations (4.1), (4.2) and (4.5) give

$$
y_{B}(t+1)=y_{B}(t)+b_{0} \Delta u(t)+b_{1} \Delta u(t-1)+w(t+1)
$$

where

$$
\begin{array}{r}
\begin{aligned}
w(t+1)=e_{y}(t+1)-e_{y}(t)+b_{0} e_{v}(t)+b_{1} e_{v}(t-1) & +b_{0} e_{u}(t) \\
& -\left(b_{0}-b_{1}\right) e_{u}(t-1)-b_{1} e_{u}(t-2)
\end{aligned}
\end{array}
$$

According to the spectral factorization theorem the disturbance $w(t)$ can be written in terms of a stable linear filter driven by white noise ( $\AA$ ström 1970),

$$
w(t+1)=e(t+1)+c_{1} e(t)+c_{2} e(t-1)
$$

where $E e(t)^{2}=\sigma^{2}$. The parameters $c_{1}, c_{2}$ and $\sigma$ are obtained from the autocovariance function

$$
\left.\begin{array}{rl}
r(0)=E w(t)^{2} & =2 \sigma_{y}{ }^{2}+\left(b_{0}{ }^{2}+b_{1}{ }^{2}\right) \sigma_{v}{ }^{2}+2\left(b_{0}{ }^{2}+b_{1}{ }^{2}-b_{0} b_{1}\right) \sigma_{u}{ }^{2} \\
& =\sigma^{2}\left(1+c_{1}{ }^{2}+c_{2}{ }^{2}\right) \\
r(1)=E w(t) w(t-1) & =-\sigma_{y}{ }^{2}+b_{0} b_{1} \sigma_{v}{ }^{2}-\left(b_{0}-b_{1}\right)^{2} \sigma_{u}{ }^{2} \\
& =\sigma^{2}\left(c_{1}+c_{1} c_{2}\right) \\
r(2)=E w(t) w(t-2) & =-b_{0} b_{1} \sigma_{u}{ }^{2}=\sigma^{2} c_{2} \\
r(3)=r(4) & =\ldots=0
\end{array}\right\}
$$


The solution to these equations is not unique, but the solution for which the zeros of the polynomial

are outside the unit circle is

$$
1+c_{1} z+c_{2} z^{2}
$$

$$
c_{1}=-0 \cdot 58, \quad c_{2}=-0 \cdot 27, \quad \sigma^{2}=43
$$

The final model relating the measured LSF at point $B$ to the desired LSF of the total feed is thus

$$
y_{B}(t+1)=y_{B}(t)+b_{0} \Delta u(t)+b_{1} \Delta u(t-1)+e(t+1)+c_{1} e(t)+c_{2} e(t-1)
$$

The dynamics of the homogenization silos can approximtely be described by a first-order system with time delay (Nyman 1976). If it is assumed that the silo input is constant during the sampling period, the system may be sampled. The sampled model for the silo dynamics is

$$
y^{\prime}(t+L+1)=\alpha y^{\prime}{ }_{c}(t+L)+\beta y_{B}^{\prime}(t)
$$

In the present investigation, the following numerical values are used:

$$
\alpha=0.93 \text { and } \beta=0.07
$$

which correspond to a time constant $T \approx 14$ hours. The time delay $L$ is varying in the region (10-20) hours owing to different operating conditions of the homogenization silos.

\section{Simulations}

Extensive simulations were carried out in order to evaluate the performance of different regulators for the cement raw material mixing process. Specifically, the performance of the self-tuning regulator, described in $\S 2$, was investigated. In the simulations, the process was modelled by eqns. $(4.1-4.4)$ and (4.11).

A simulation of the uncontrolled system is shown in Fig. 2. The desired input $u$ to the ball mill was, in this simulation, held equal to zero. The simulation shows that the disturbances pass the ball mill undamped. The homogenization silos damp high frequencies effectively, whereas low frequencies pass undamped. A successful control of the system should, therefore, be able to damp low frequency disturbances.

\section{Minimum variance after ball mill}

Firstly, regulators designed to minimize the variance of the ball mill output $y_{B}^{\prime}$ are considered. From eqn. (4.2) it follows that

$$
E y_{B}^{\prime}(t)^{2}=E y_{B}(t)^{2}-\sigma_{y}^{2}
$$

where $\sigma_{y}{ }^{2}$ is the variance of the measurement error. It is thus, equivalent to find a strategy which minimizes the variance of the measured output $y_{B}$.

The minimum variance strategy for the system described by $(4.10)$ is

$$
\begin{aligned}
\Delta u(t) & =-\frac{1+c_{1}}{b_{0}} y_{B}(t)-\frac{c_{2}}{b_{0}} y_{B}(t-1)-\frac{b_{1}}{b_{0}} \Delta u(t-1) \\
& =-0.56 y_{B}(t)+0.36 y_{B}(t-1)-0.32 \Delta u(t-1)
\end{aligned}
$$




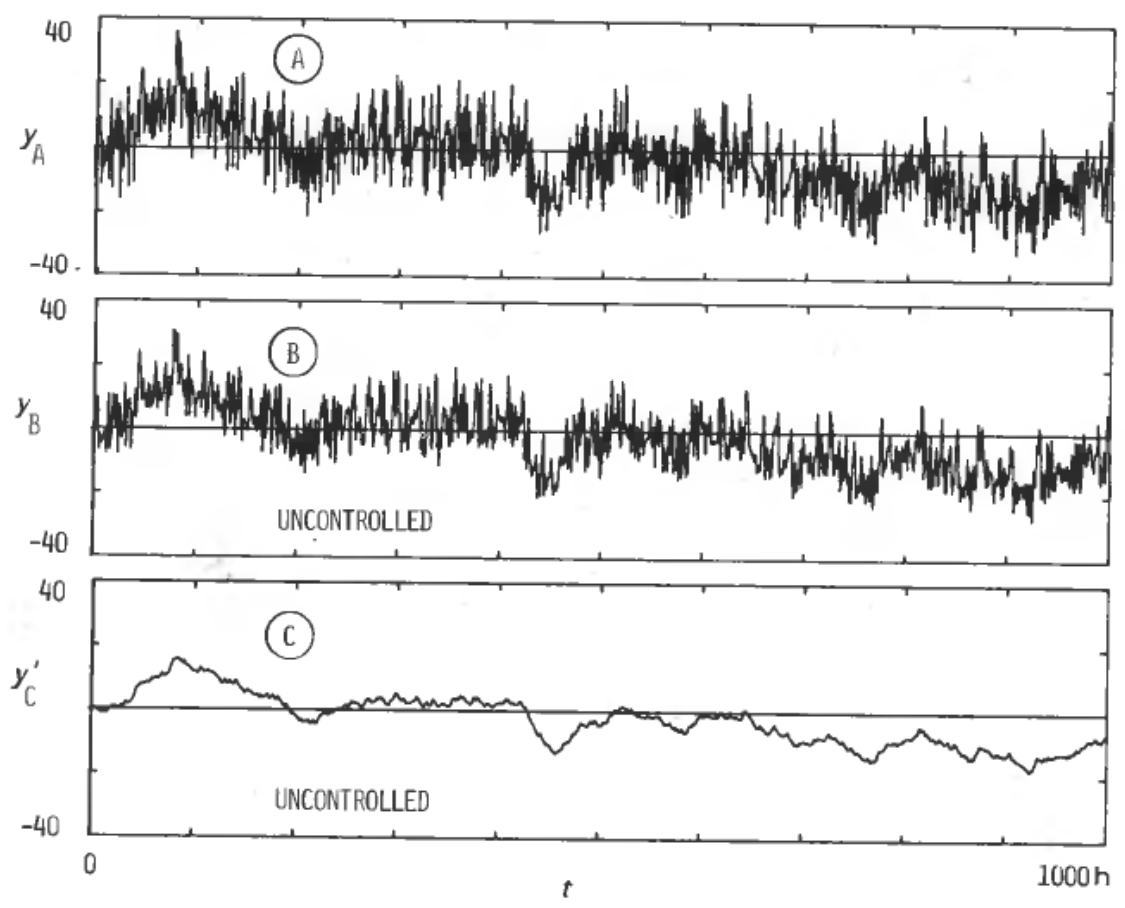

Figure 2. Simulated response of the uncontrolled mixing system.

For this strategy, the ball mill output is asymptotically a white-noise sequence with variance

$$
E y_{B}(t)^{2}=\sigma^{2}=43
$$

and

$$
E y_{B}^{\prime}(t)^{2}=\sigma^{2}-\sigma_{y}^{2} \simeq 42
$$

The output from the homogenization silos will be a drifting process with variance

$$
\begin{aligned}
E y_{c}^{\prime}(t+L+1)^{2} & =E\left[\sum_{s=-\infty}^{t} \beta \alpha^{t-s} y_{B}^{\prime}(s)\right]^{2} \\
& =\beta^{2}\left(\sigma^{2}-\sigma_{y}{ }^{2}\right) /\left(1-\alpha^{2}\right) \simeq 1 \cdot 5
\end{aligned}
$$

Figure 3 shows the result when the strategy (5.1) was simulated for the mixing system. Control of the ball mill output is good but the silo output is still drifting. It is obvious that this strategy is not well suited for control of the silo output.

\section{Minimum variance after silos}

The object of the mixing system is to minimize the variance of $y_{c}^{\prime}$, the output from the homogenization silos. However, owing to the long and time-varying time delay of the silos, it is not possible in practice to use measurements of $y_{c}$ for feedback. Therefore, an estimate of $y_{c}$ is used instead. From the knowledge of the silo dynamics discussed in $\S 4$, an estimate of the silo output is given by

$$
\hat{y}_{c}(t+L+1)=\alpha \hat{y}_{C}(t+L)+\beta y_{B}(t)
$$



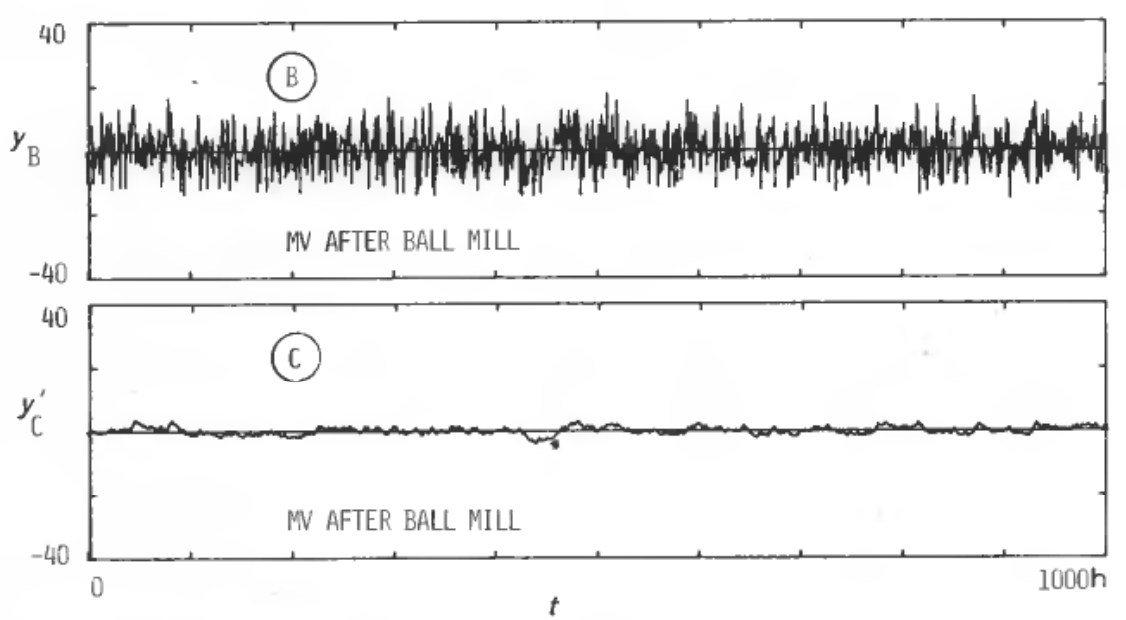

Figure 3. Simulated response of the mixing system when using the strategy (5.1) for minimum variance after ball mill.

Combining eqns. (4.10) and (5.2) gives

$$
\begin{aligned}
\hat{y}_{c}(t+L+2)=(1+\alpha) \hat{y}_{c}(t+L & +1)-\alpha \hat{y}_{c}(t+L)+\beta b_{0} \Delta u(t) \\
& +\beta b_{1} \Delta u(t-1)+\beta\left[e(t+1)+c_{1} e(t)+c_{2} e(t-1)\right]
\end{aligned}
$$

and the strategy minimizing $E \hat{y}_{c}(t)^{2}$ is

$$
\begin{aligned}
\Delta u(t) & =-\frac{1+\alpha+c_{1}}{\beta b_{0}} \hat{y}_{c}(t+L+1)-\frac{c_{2}-\alpha}{\beta b_{0}} \hat{y}_{c}(t+L)-\frac{b_{1}}{b_{0}} \Delta u(t-1) \\
& =-25 \cdot 4 \hat{y}_{c}(t+L+1)+22 \cdot 6 \hat{y}_{c}(t+L)-0 \cdot 316 \Delta u(t-1)
\end{aligned}
$$

It is convenient to use a control law in which only $y_{B}$ and $\Delta u$ are fed back. Multiplying (5.4) by $\left(1-\alpha q^{-1}\right)$ and using (5.2) gives

$$
\begin{aligned}
\Delta u(t) & =-\frac{1+\alpha+c_{1}}{b_{0}} y_{B}(t)-\frac{c_{2}-\alpha}{b_{0}} y_{B}(t-1)+\left(\alpha-\frac{b_{1}}{b_{0}}\right) \Delta u(t-1)+\frac{\alpha b_{1}}{b_{0}} \Delta u(t-2) \\
& =-1.78 y_{B}(t)+1.58 y_{B}(t-1)+0.614 \Delta u(t-1)+0.294 \Delta u(t-2)
\end{aligned}
$$

As the zero of $1-\alpha z$ is outside the unit circle, the control law (5.5) is asymptotically equal to (5.4).

When using the strategy (5.4) or (5.5) the estimated silo output $\hat{y}_{C}$ is asymptotically a white-noise sequence with variance

$$
E \hat{y}_{C}(t)^{2}=\beta^{2} \sigma^{2} \approx 0 \cdot 21
$$

The variance of the ball mill output will be

$$
E^{\prime}{ }_{B}(t)^{2} \approx 79
$$

which can be computed by the methods described by Åström (1970). 


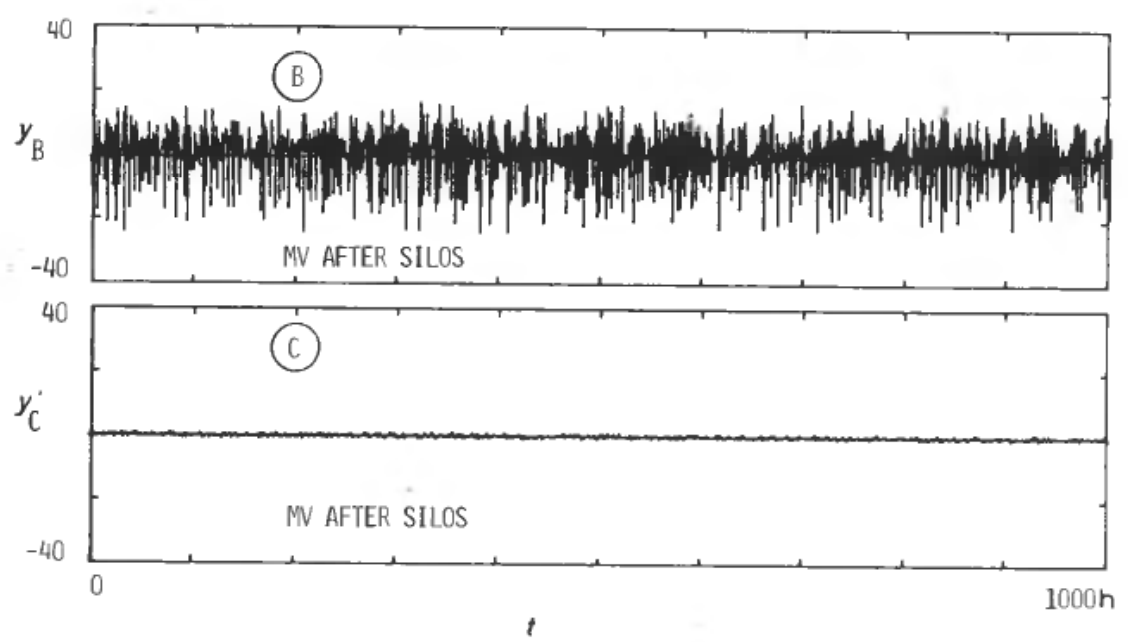

Figure 4. Simulated response of the mixing system when using the strategy (5.5) for minimum variance after silos.

The result, when the strategy (5.5) was simulated for the system, is shown in Fig. 4. A self-tuning regulator can now be based on the model

$$
\Delta u(t)=h \hat{y}_{c}(t+L+2)+K v(t)+\tilde{\epsilon}(t+L+2)
$$

and using the control

$$
\Delta u(t)=\hat{K} v(t)
$$

The table shows the performance of self-tuning regulators with different structures. Asymptotically the optimal regulator structure (1) gives the best performance. However, the Table also shows that the loss of the transient period is considerably

\begin{tabular}{|c|c|c|c|c|c|}
\hline \multirow[b]{2}{*}{ Regulator structure } & \multicolumn{4}{|c|}{$\bar{V} / \bar{V}_{\min }$} & \multirow[b]{2}{*}{$\infty$} \\
\hline & $t=100$ & 500 & 1000 & 5000 & \\
\hline (1) $\begin{aligned} \Delta u(t)= & \hat{k}_{1} \Delta u(t-1)+\hat{k}_{2} \hat{y}_{c}(t+L+1) \\
& +\hat{k}_{3} \hat{y}_{c}(t+L)\end{aligned}$ & $5 \cdot 26$ & 1.91 & $1 \cdot 49$ & $1 \cdot 12$ & $1 \cdot 00$ \\
\hline (2) $\Delta u(t)=\hat{k}_{1} y_{B}(t)+\hat{k}_{2} y_{B}(t-1)$ & $3 \cdot 29$ & $1 \cdot 54$ & $1 \cdot 38$ & $1 \cdot 19$ & $1 \cdot 11$ \\
\hline (3) $\Delta u(t)=\hat{k}_{1} \Delta u(t-1)+\hat{k}_{2} y_{B}(t)$ & $8 \cdot 65$ & $2 \cdot 55$ & $1 \cdot 82$ & $1 \cdot 18$ & $1 \cdot 02$ \\
\hline (4) $\Delta u(t)=\hat{k}_{1} y_{B}(t)+\hat{k}_{2} \hat{y}_{C}(t+L+1)$ & $1: 69$ & $1 \cdot 64$ & $1 \cdot 59$ & $1 \cdot 58$ & $1 \cdot 62$ \\
\hline (5) $\Delta u(t)=\hat{k}_{1} \hat{y}_{c}(t+L+1)+\hat{k}_{2} \hat{y}_{c}(t+L)$ & $1 \cdot 64$ & $1 \cdot 57$ & $1 \cdot 57$ & $1 \cdot 58$ & $1 \cdot 62$ \\
\hline (6) $\Delta u(t)=\hat{k}_{1} \Delta u(t-1)+\hat{k}_{2} \hat{y}_{c}(t+L+1)$ & 6.83 & $3 \cdot 22$ & $2 \cdot 81$ & $2 \cdot 39$ & $2 \cdot 42$ \\
\hline (7) $\Delta u(t)=\hat{k}_{1} y_{B}(t)$ & $1 \cdot 82$ & $1 \cdot 45$ & $1 \cdot 39$ & $1 \cdot 35$ & $1 \cdot 38$ \\
\hline
\end{tabular}
smaller when using regulators with only two estimated parameters. As the dynamics

Simulation of self-tuning regulators based on (5.6) for minimizing the variance of the silo output. The regulators were applied with $\hat{k}_{\mathrm{i}}(0)=0, h=20, \boldsymbol{P}(0)=I$, $\lambda=0.99$. The accumulated loss is $V=\sum_{s=0}^{t} y_{c}^{\prime}(s)^{2}$ and $V_{\min }$ is the accumulated loss when using the optimal strategy (5.4) or (5.5). The values in the table represent the mean of ten simulations. The asymptotic loss was calculated by methods described by Åström and Wittenmark (1973). 
of the process can be expected to change with time, and as the sampling interval is long ( 1 hour), it is important to have a regulator with good transient properties, which can adapt to changing process dynamics in only a few samples. The simulations, therefore, indicate that it is doubtful whether anything can be gained by using a regulator in which more than two parameters are estimated. The result, when the selftuning regulator (2) of the Table was simulated for the mixing system, is shown in Fig. 5 .
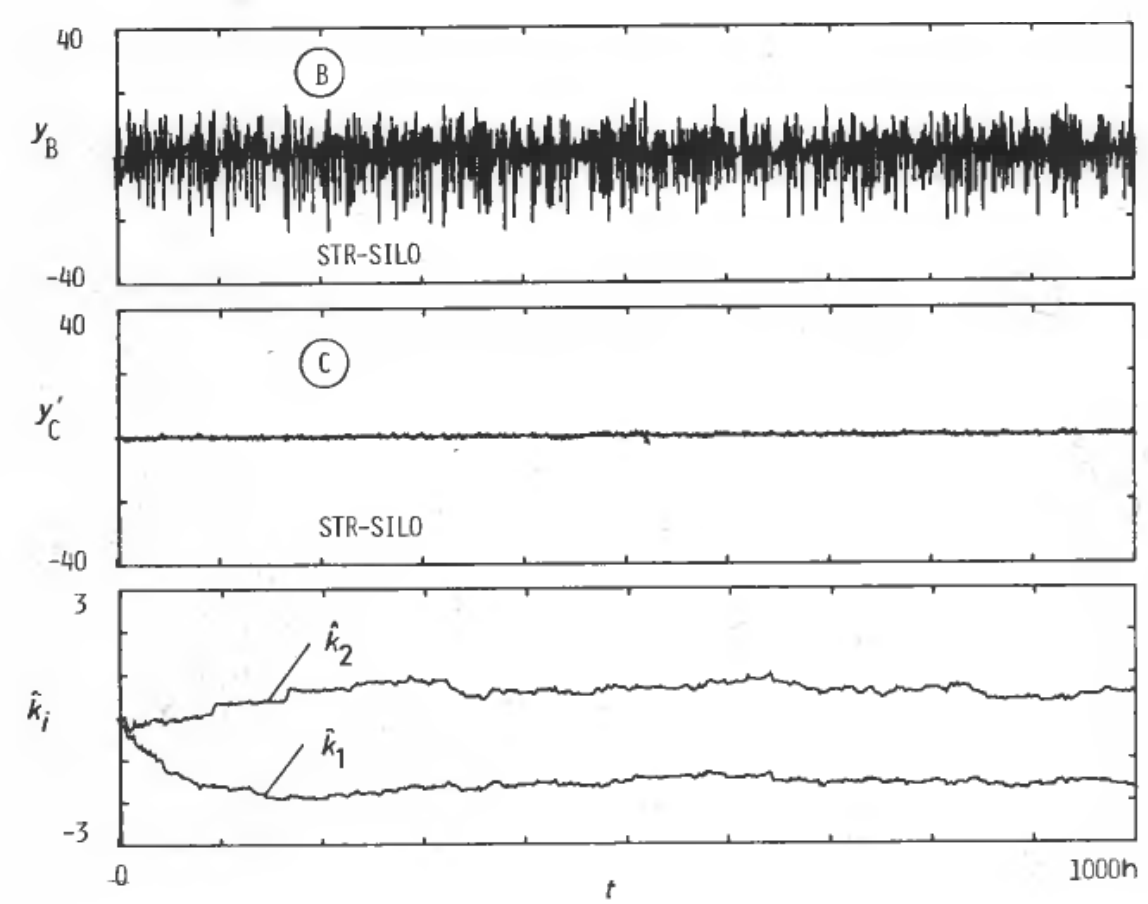

Figure 5. Simulated response of the mixing system when using the self-tuning regulator (2) of the Table for minimum variance after silos.

As the regulator uses a model of the silo dynamics, it is relevant to investigate how sensitive the performance of the regulator is to the choice of the silo model. This can be done by calculating the variance of $\hat{y}_{C}$ for different time constants $T_{s}$ for the silo when using, (i) the optimal strategy which can be calculated when $T_{s}$ is known, and (ii) the strategy (5.4) based on $T_{s} \approx 14$ hours $(\alpha=0.93)$. It turns out that the performance of the strategy (5.4) remains close to the optimal even for large variations of the time constant $T_{s}$. Thus, for $T_{s}=3$ hours the optimal strategy gives the variance $3 \cdot 5$ whereas the strategy (5.4) gives the variance $3 \cdot 8$, and for $T_{s}=30$ hours the corresponding variances are 0.046 and 0.047 . It can thus be concluded that the choice of the silo model is not a critical step in the design of a regulator for the system.

\section{Comparison with other strategies}

It is of interest to compare the performance of the minimum variance strategies and the self-tuning regulators with other strategies, which have previously been considered for the mixing system. 
The process was originally controlled by the digitalized PI-regulator

$$
\Delta u(t)=-0 \cdot 65 y_{B}(t)+0 \cdot 5 y_{B}(t-1)
$$

Hammarström et al. (1978) have attacked the control problem using deterministic control theory. Two regulators which were considered by Hammarström et al. (1978) are

$$
\begin{gathered}
\Delta u(t)=-0.94 y_{B}(t)+0.02 y_{B}(t-1)-0.30 \Delta u(t-1) \\
\Delta u(t)=-y_{B}(t)+0.08 y_{B}(t-1)-0.20 \Delta u(t-1)
\end{gathered}
$$

The control law (5.8) was derived using optimal deterministic control theory, and the strategy (5.9) is a Higham controller (Higham 1968) for time delay systems. The strategy (5.9) was found to give better control of the silo output than the original PI-regulator, and the strategy has, therefore, been implemented in the process.

The regulators (5.7-5.9) were simulated for the mixing system. The accumulated loss

$$
V=\sum_{s=0}^{t} y(s)^{2}
$$

of the ball mill output and the silo output is shown in Figs. 6 and 7 when using different strategies. It is interesting to observe that the original PI-regulator is well tuned for minimizing the variance of the ball mill output, but it does not give good performance at the silo output.

\section{Experimental results}

The self-tuning regulator considered in $\S 5$ was implemented in the cement raw material mixing system of Oy Partek Ab. It was decided to use a regulator in which only two parameters are estimated. The simulations of $\S 5$ indicated that probably nothing could be gained by estimating more parameters. A priori knowledge was included in the regulator by assuming that the deterministic part of the system is constant and known. Then only those parameters which depend on the stochastic part of the system were estimated. Several regulator structures were tried. The results were very encouraging and two examples are given below. An example showing the result when a regulator with fixed parameters was used is also given.

The performance of the regulators was evaluated by calculating the variances of the ball mill output and the estimated silo output. The actual silo output was, in these experiments, disturbed by a side flow of dust of varying size from electric precipitators which could not be compensated for. The variance of the silo output is, therefore, not given.

\section{Example 1 (November 1977)}

This example shows the result when using a regulator with fixed parameters. The strategy given by eqn. (5.9) was used. Figure 8 shows the ball mill output $Y_{B}$, the estimated silo output $\hat{Y}_{C}$, and the measured silo output $Y_{C}$ for this example. Observe that the measured silo output, $Y_{c}$, is directly affected by the measurement noise $(\sigma=0.9)$ contrary to the estimate of the silo output, $\hat{Y}_{C}$. Furthermore, the mean and the 


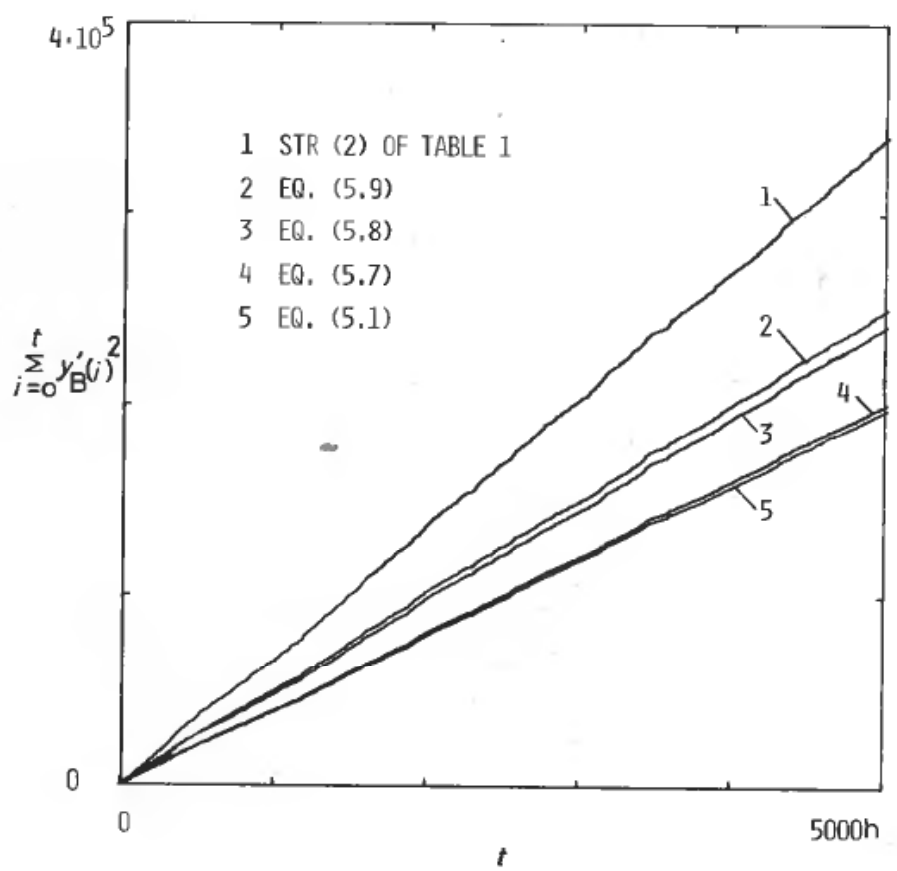

Figure 6. Accumulated loss of the ball mill output when using different regulators.

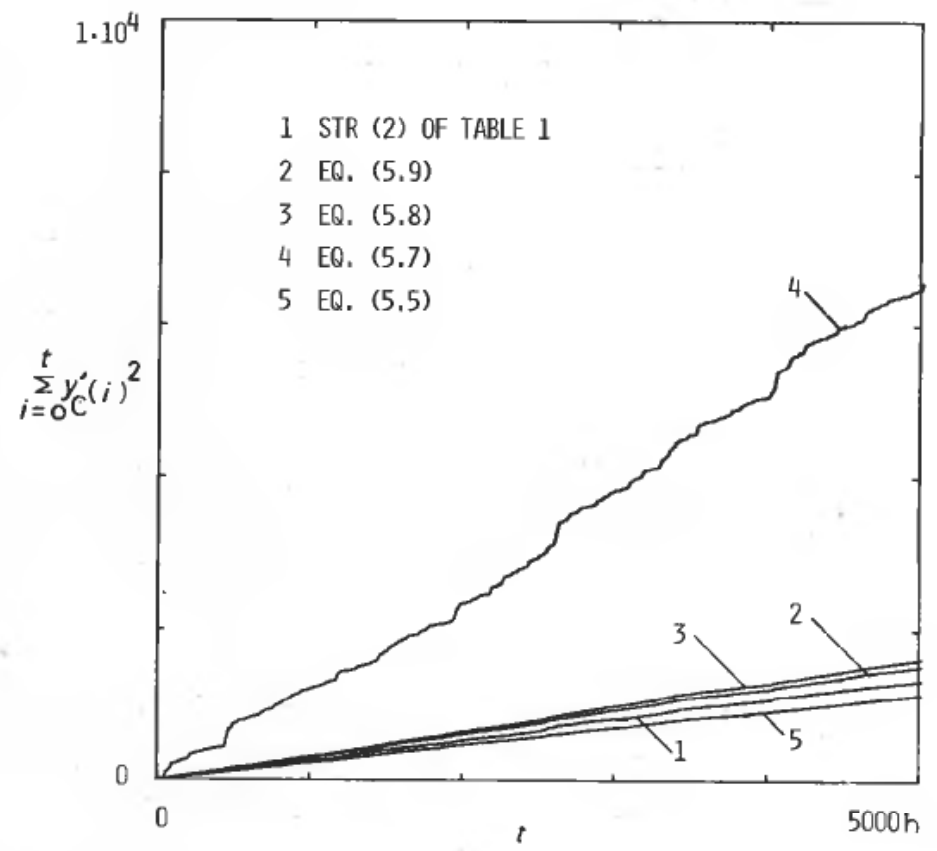

Figure 7. Accumulated loss of the silo output when using different regulators. 


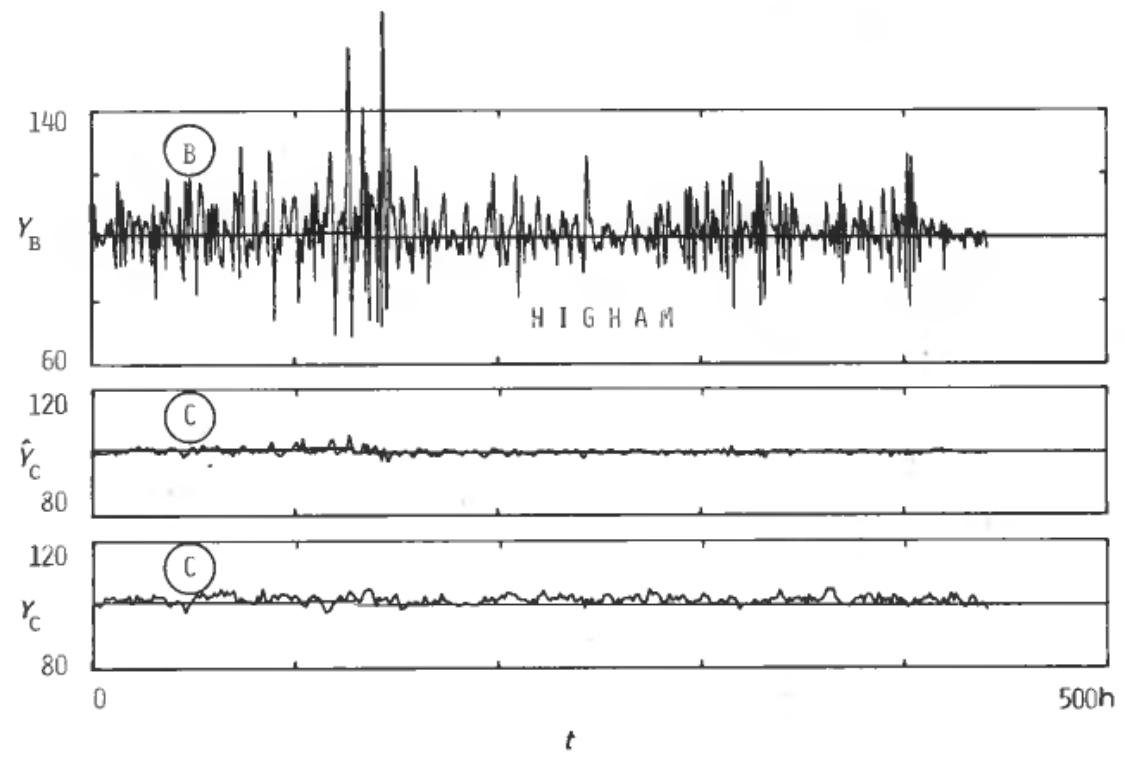

Figure 8. Results from Example 1. The silo output $Y_{c}$ was disturbed by a side flow of dust from electric precipitators which could not be compensated for.

variance of the measured silo output, $Y_{C}$, is affected by the side flow of dust from electric precipitators. The variance of the ball mill output was

$$
s_{y_{B}}^{2}=\frac{1}{N} \sum_{i=1}^{N} y_{B}(i)^{2}=116
$$

and the variance of the estimated silo output was

$$
s_{\hat{y} c}^{2}=\frac{1}{N} \sum_{i=1}^{N} \hat{y}_{c}(i)^{2}=0 \cdot 77
$$

\section{Example 2 (June 1978)}

In this example, two regulator parameters were estimated, and a third was assumed to be known. Using eqn. (5.2) the strategy (5.4) can be written

$$
\Delta u(t)=k_{1} y_{B}(t)+k_{2} \hat{y}_{C}(t+L+1)+k_{3} \Delta u(t-1)
$$

where

$$
k_{1}=-\frac{\alpha-c_{2}}{\alpha b_{0}}, \quad k_{2}=-\frac{\alpha^{2}+\alpha c_{1}+c_{2}}{\alpha \beta b_{0}}, \quad k_{3}=-\frac{b_{1}}{b_{0}} \approx-0.32
$$

Here the parameter $k_{3}$ is determined by the ball mill dynamics, eqn. (4.1), which is well known from experiments. The parameters $k_{1}$ and $k_{2}$ depend, through $c_{1}$ and $c_{2}$, on the characteristics of the disturbances, which are less well known and may change with time. It therefore seems reasonable to use a self-tuning regulator in which only $k_{1}$ and $k_{2}$ are estimated and the parameter $k_{3}$ is assumed to be known. 
In this example, the parameters $k_{1}$ and $k_{2}$ were estimated from the model

$$
\Delta u(t)=h \hat{y}_{C}(t+L+2)-0 \cdot 32 \Delta u(t-1)+k_{1} y_{B}(t)+k_{2} \hat{y}_{C}(t+L+1)
$$

and the control signal was determined from

$$
\Delta u(t)=-0 \cdot 32 \Delta u(t-1)+\hat{k}_{1} y_{B}(t)+\hat{k}_{2} \hat{y}_{c}(t+L+1)
$$

The regulator parameters were

$$
h=20 \cdot 0 \text { and } \lambda=0.96
$$

The result is shown in Fig. 9. The variances of the ball mill output and the estimated silo output were

$$
s_{y_{B}}{ }^{2}=59.0 \text { and } s_{\varphi_{c}}{ }^{2}=0.51
$$

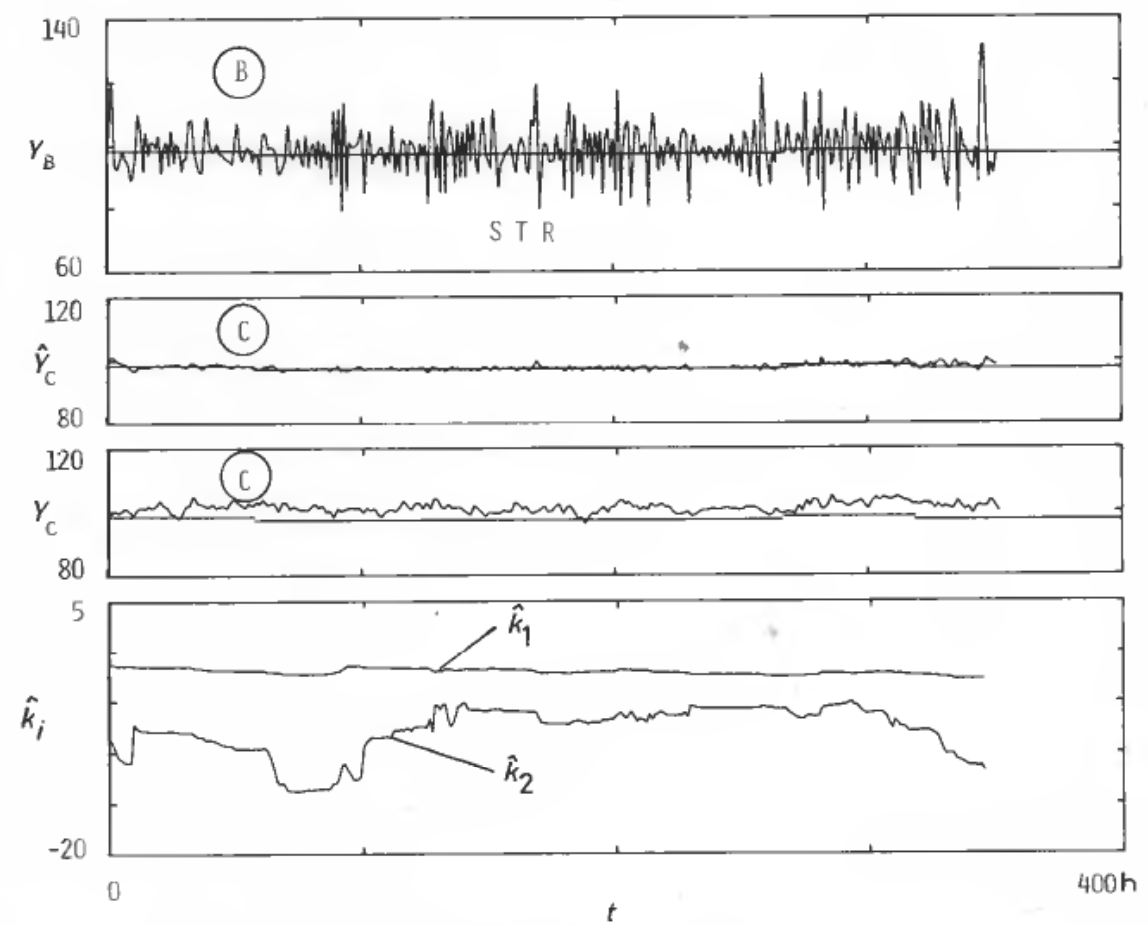

Figure 9. Results from Example 2. The silo output $Y_{C}$ was disturbed by a side flow of dust from electric precipitators which could not be compensated for.

\section{Example 3 (August 1978)}

In this example, the regulator structure (5.4) was used. Again, it was assumed that the parameters $b_{0}$ and $b_{1}$ are known. The regulator was thus

$$
\Delta u(t)=-0 \cdot 32 \Delta u(t-1)+\hat{k}_{1} \hat{y}_{c}(t+L+1)+\hat{k}_{2} \hat{y}_{c}(t+L)
$$

where $k_{1}$ and $k_{2}$ were estimated. The regulator parameters were

$$
h=20.0 \text { and } \lambda=0.99
$$




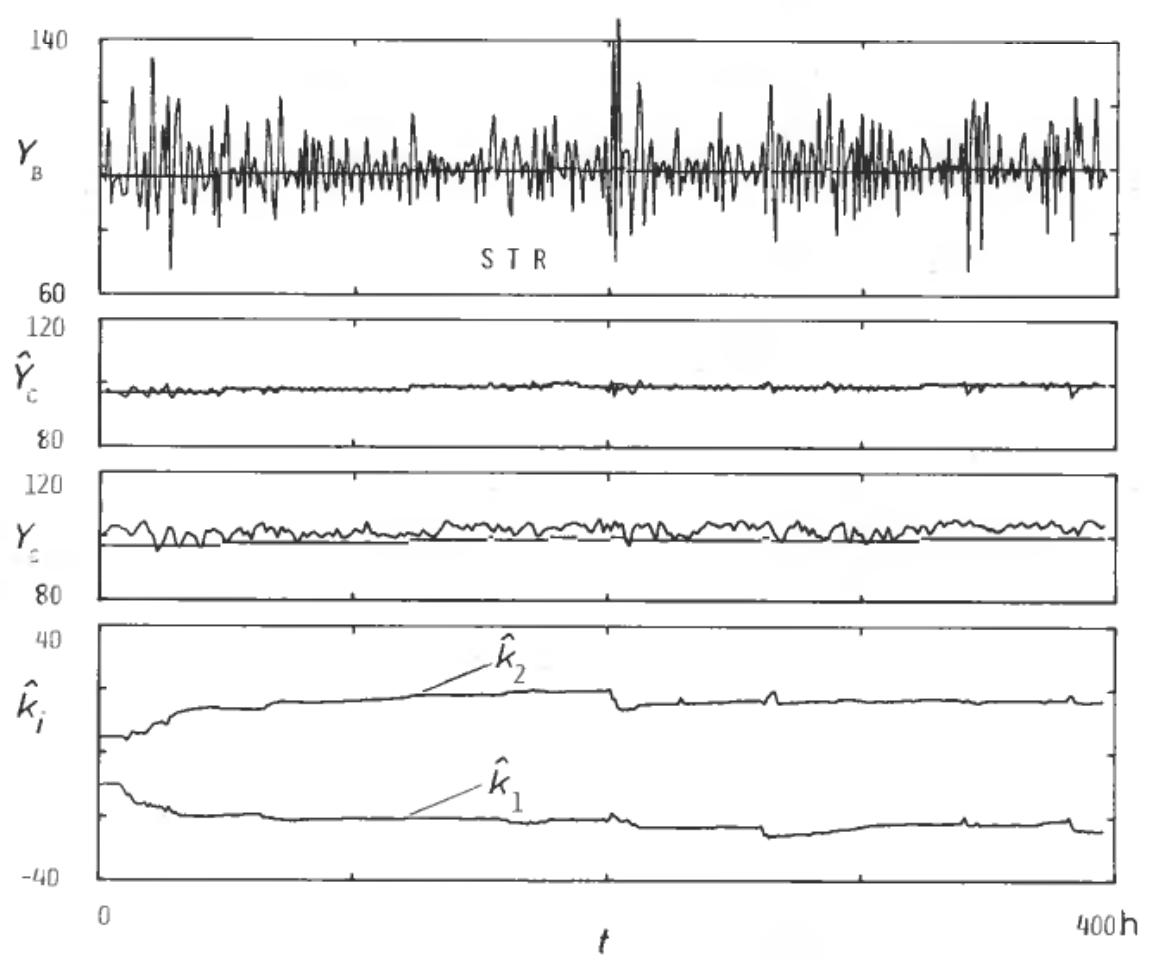

Figure 10. Results from Example 3. The silo output $Y_{C}$ was disturbed by a side flow of dust from electric precipitators which could not be compensated for.

The result is shown in Fig. 10. The variances in this example were

$$
s_{y_{B}}{ }^{2}=104 \cdot 0 \text { and } s_{\hat{j} c^{2}}=0.57
$$

Figure 11 shows the trajectory of the parameter estimates. The shaded area shows the region in which the estimates were during the practical experiment. Level curves for the ratio (variance after silo)/(minimum variance after silo) calculated for the system model (5.3) are also shown (cf. Westerlund 1979). The figure shows that the regulator parameters were quite close to the optimal parameters for the system model (5.3), during the experiment.

The experience obtained from practice has been that the self-tuning regulator, on average, gives better control performance than a regulator with carefully tuned fixed parameters. As it is difficult to tune the regulator parameters manually, especially when the process dynamics are changing, it is clearly advisable to use a self-tuning regulator. After the first practical experiments had been performed, the self-tuning regulator used in Example 3 above has been in continuous use.

\section{Conclusions}

Stochastic modelling and control of a continuously operating cement raw material mixing system have been considered. A special feature of the control problem is the fact that measurements of the controlled variable, the composition of the silo output, cannot be used for feedback owing to the long and changing time delay of the silos. Instead, the composition of the silo output is estimated from measurements of the 


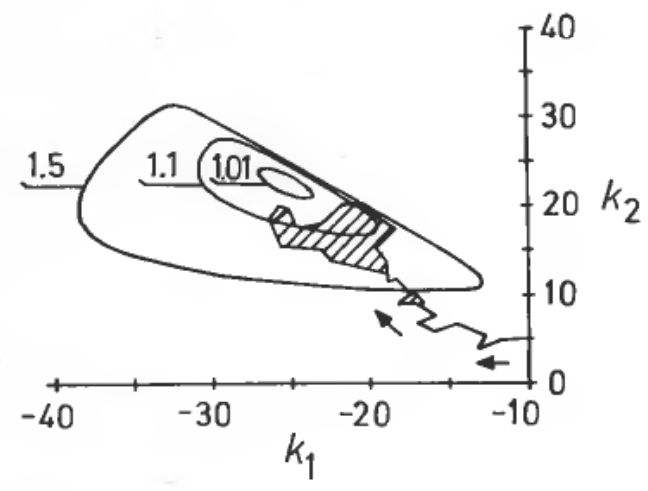

Figure 11. Phase plane of the regulator parameters in Example 3. The shaded area shows the region in which the parameters were during the practical experiment. Level curves for the ratio $E \hat{y}_{c}(t)^{2} / \mathrm{min} E \hat{y}_{c}(t)^{2}$ calculated for the system model (5.3) are also shown.

silo input, using a first-order model for the silo dynamics. The regulator is then designed for minimizing the variance of the estimated silo output. The performance of the regulator is not sensitive to the choice of the silo model when the silos are sufficiently large.

The feasibility of the self-tuning regulator for control of the mixing system was investigated by simulations. From the simulations it was concluded that, for the system under study, it is preferable to use a self-tuning regulator in which a fewer number of parameters is estimated than the optimal regulator would require. This was motivated by the superior transient properties of the simpler regulator in combination with the large sampling interval (1 hour).

Finally, results from the implementation of the self-tuning regulator are given. The results show once more the practical applicability of the self-tuning regulator. The control performance was so good that the regulator has been permanently installed in the system,

\section{ACKNOWLEDGMENTS}

Financial support from the Foundation of Neste OY to two of us (T.W. and H.T.) is gratefully acknowledged. The authors would also like to express their gratitude to Oy Partek Ab for providing the opportunity to implement the regulators.

\section{REFERENCES}

Åström, K. J. (1970). Introduction to Stochastic Control Theory (Academic Press).

Åström, K. J., Borisson, U., LjUNG, L., and WitTenmark, B. (1977). Theory and applications of self-tuning regulators. Automatica, 13, 457-476.

Åström, K. J., and WitTenmark, B. (1973). On self-tuning regulators. Automatica, 9, $185-199$.

Borisson, U., and Syding, R. (1976). Self-tuning control of an ore crusher. Automatica, $12,1-7$.

Borisson, U., and WitTENMARK, B. (1974). An industrial application of a self-tuning regulator. 4th IFAC Symposium on Digital Computer Applications to Process Control, Zürich, Switzerland.

Cegrell, T., and Hedqvist, T. (1975). Successful adaptive control of paper machines. Automatica, 11, 53-59.

ЕукноғF, Р. (1974). System Identification (Wiley). 
Hammarström, L. G., Nyman, K.-E., Gros, K. S., and Waller, K. V. T. (1978). Optimal computer control of a cement raw mill-a case study. Kemia-Kemi, 5, 208-211.

Hammer, H. (1972). Computer controlled raw meal production in cement industry. Regelungstechnik, 20, 190-198.

Higham, J. D. (1968). Single-term control of first- and second-order processes with dead time. Control, 12, 136-140.

Jensen, L., and HANSEL, R. (1974). Computer control of an enthalphy exchanger. Report 7417. Department of Automatic Control, Lund Institute of Technology, Sweden.

KeviczKy, K., Hetthessy, J., Hilger, M., and Kolostori, J. (1978). Self-tuning adaptive control of cement raw material blending. Automatica, 14, 525-532.

Källström, C. G., Åström, K. J., Thorell, N. E., Eriksson, J., and Sten, L. (1978). Adaptive autopilots for steering of large tankers. IFAC Congress, Helsinki, Finland.

LAURÉN, K.-G. (1978). Raw meal preparation-a new technological system. 13th International Cement Seminar, Rock Products, Chicago, U.S.A.

LundÁN, A., and MATTILA, O. (1974). A system for the control of the homogenization of the cement raw meal. 4th IFAC Symposium on Digital Computer Applications to Process Control, Zürich, Switzerland.

Nyman, K.-E. (1976). Computer control of cement raw material mixing (in Swedish). Report OY Partek Ab, Pargas, Finland.

Sastry, V. A., Seborg, D. E., and Wood, R. K. (1977). Self-tuning regulator applied to a binary distillation column. Automatica, 13, 417-424.

ToIVONEN, H. (1977). Self-tuning minimum variance control of multivariable systems. Report 77-6, The Institute for Automatic Control, Åbo Akademi, Finland.

Westerlund, T. (1979). Sensitivity and optimal tuning of linear time-invariant controllers with a stationary quadratic loss function. Report 79-18-W, Division of Engineering Cybernetics, The Norwegian Institute of Technology, Norway. 